P04-1-90 Poster session

\title{
Cell-type-specific targeting of viral vectors in the mammalian brain
}

\author{
Toshiaki Suzuki ${ }^{1}$, Yuji Masaki ${ }^{1}$, Taiki Hara ${ }^{1}$, Nao Morimoto ${ }^{1,2}$, Fumitaka Osakada ${ }^{1,2,3}$ \\ ${ }^{1}$ Laboratory of Cellular Pharmacology, Graduate School of Pharmaceutical Sciences, Nagoya University, Japan, \\ ${ }^{2}$ Laboratory of Neural Information Processing, Institute for Advanced Research, Nagoya University, Japan, ${ }^{3}$ PRESTO, \\ Japan Science and Technology Agency, Japan
}

The function of the nervous system arises from complex interactions between networks of neurons composed of multiple cell types. Understanding cell-type-specific connectivity at the whole brain level will be required for understanding normal brain function and neurological disorders. G-deleted rabies viral vectors (RVdG) has been used for revealing neuronal connectivity in the mammalian brain. To understand interaction of complex neural circuits, we sought to visualize and manipulate multiple distinct populations with RVdG. Here we developed viral targeting systems: EnvB/TVB and EnvE/DR46-TVB systems and bridge-protein-mediated system.

The interaction between the envelope protein of a virus and cell-surface receptors in a recipient cell leads to viral infection onto a target cell. Viruses whose envelope protein is EnvA derived from avian sarcoma and leukosis viruses bind to its receptor TVA. Pseudotyping of envelope viruses including RVdG and HIV vectors with the foreign envelope EnvA allows us to introduce fluorescent proteins or optogenetic tools in cells of interests without any infection of other cells in the mammalian brain. But in addition to EnvA/TVA system, other specific infection systems are required for labeling two distinct populations. EnvB/RV-G or EnvE/RV-G chimeric glycoprotein was generated for pseudotyping of RVdG with EnvB or EnvE, respectively. Envelope-specific receptors TVB and DR46-TVB were optimized and fused with a fluorescent protein for visualization of receptor-expressing cells. Combination of EnvB/TVB and EnvE/DR46-TVB systems with EnvA/TVA system allows for simultaneous targeting of RVdG to three distinct cell populations.

Cre-dependent transduction to cell types that selectively express Cre recombinase in transgenic animals is the most powerful to introduce TVA, TVB or DR46-TVB in a specific cell population. In contrast, for targeting a specific cell type in non-transgenic animals, bridge-protein-mediated transduction allows cell-type-specific virus infection based on the expression of surface proteins including receptors. A bridge protein composed of TVB fused to Neuregulin can be used for targeting an ErbB4-expressing subtype of inhibitory neurons in non-transgenic animals. We established an efficient strategy to identify more presynaptic neurons to ErbB4-expressing inhibitory neurons.

The viral targeting strategies will be powerful to dissect complex neural circuits, providing better opportunities to address circuit-level questions in health and neurological diseases. 